\title{
Visible diode lasers for high bitrate underwater wireless optical communications
}

\author{
Boon S. Ooi, ${ }^{1,3}$ Xiaobin Sun, ${ }^{1}$ Omar Alkhazragi, ${ }^{1}$ Yujian Guo, ${ }^{1}$ Tien Khee Ng, ${ }^{1}$ Mohamed-Slim Alouini ${ }^{2}$ \\ ${ }^{\text {I}}$ Photonics Laboratory, King Abdullah University of Science \& Technology (KAUST), Thuwal 23955-6900, Saudi Arabia \\ ${ }^{2}$ Communication Theory Laboratory, King Abdullah University of Science \& Technology (KAUST), Thuwal 23955-6900, Saudi Arabia \\ 3boon.ooi@kaust.edu.sa
}

\begin{abstract}
This talk provides an overview of the latest underwater wireless optical communication (UWOC) research from the system to the device level. Besides, studies investigating underwater channel characterization are also described.

OCIS codes: (060.4510) Optical communications; (250.0250) Optoelectronics; (040.5160) Photodetectors.
\end{abstract}

Numerous human underwater activities, ranging from mineral resource exploiting to marine life discovery and even environment monitoring, creating huge demand of highly reliable, secured and high data rate underwater wireless communication systems, as shown in Fig. 1. Compared with radio-frequency (RF) and acoustic wave underwater communications, underwater wireless optical communications (UWOC) outperform in various aspect, such as unlicensed spectrum, large bandwidth (THz) [1] and low data latency.

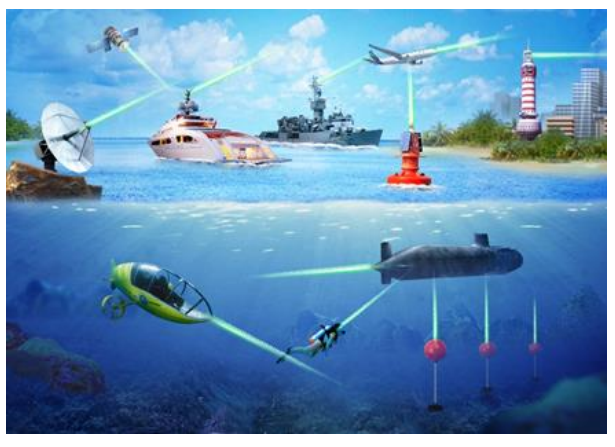

Fig. 1. Illustration of underwater human activities [11].

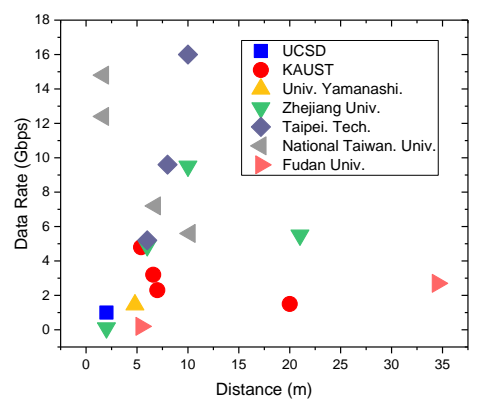

Fig. 2. Advances of recent UWOC.

The first investigation on UWOC was proposed by Karp in 1970's, who characterized channel between underwater and satellite utilizing multiple scattering model [2]. In 1992, using an argon-ion $\left(\mathrm{Ar}^{+}\right)$514-nm laser, Snow et al. have experimentally demonstrated the first UWOC link with a data rate of 50 Mbps over $5.1 \mathrm{~m}$ attenuation length [3]. The first gigabit (1 Gbps) UWOC link was achieved by Hasan et al. in 2008 [4]. In that demonstration, an externally modulated and frequency doubled 532-nm laser was used to send the signal over a 2-m water tank. Recently, high speed, power efficient opto-electronic devices are utilized in realizing even tens of gigabits UWOC links. This includes light emission diodes (LEDs) [5], laser diodes [6], photodetectors (PDs) [7], amplifiers and even integration of these devices [8]. Oubei et al. demonstrated a 2.3-Gbps, 7-m UWOC link with a directly modulated 520-nm laser diode [9]. To achieve higher data rate, some more spectrum efficient modulation technique, such as orthogonal frequency division multiplexing (OFDM) and pulse amplitude modulation (PAM), are adopted in UWOC in the last few years. Kong et al. have experimentally achieved a 9.51-Gbps, 10-m UWOC link by employing 32-quadrature amplitude modulation (QAM) OFDM modulation schemes. Besides, a 450-nm blue GaN laser diode modulated by pre-leveled 16-QAM OFDM was employed, implementing a UWOC link with data rate up to 12.4 Gbps over $1.7 \mathrm{~m}$ [1]. Furthermore, Huang et al. have established filtered multicarrier 16-QAM OFDM UWOC link, achieving a data rate of $14.8 \mathrm{Gbps}$ and a transmission distance of $1.7 \mathrm{~m} \mathrm{[6]}$. Beside the OFDM, a data rate of 16 Gbps UWOC system over $10 \mathrm{~m}$ transmission distance was implemented with the PAM-4 by Liu et al in 2016 [10]. While pursuing high data rate UWOC, by taking advantages of low seawater absorption in visible portion of electromagnetic (EM) waves, these UWOC links are even demonstrated to have transmission distance over tens of meters underwater. Shen et al. achieved the first Gbps UWOC link over $20 \mathrm{~m}$ with a directly modulated 450-nm laser diode [11]. Based on this study, a 21-m, 5.5 Gbps UWOC link with OFDM modulation was further demonstrated by Chen et al. in 2017 [12]. The longest distance with a Gbps data rate UWOC system was demonstrated by Liu et al., who used a directly modulated 520-nm laser diode and a transmission distance of $34.5 \mathrm{~m}$ 
was achieved [13]. The recent advances of UWOC based on the visible light were summary in Fig. 2 [4-6, 9-21]. Besides these data transmission underwater, a high quality video streaming was achieved in real ocean water [22].

However, realistic UWOC performance should take into account the various practical scenarios in actual undersea environment, which will alter the light propagation characteristics in water. These factors include microscopic particulates suspended in various ocean waters, turbulence resulted from the change in refractive index introduced either by temperature or by salinity gradient, bubbles, etc. In the presence of microscopic particulates suspension and dissolve organic matters in different ocean waters, absorption and multiple scattering process will cause irreversible loss of optical intensity and severe temporal pulse broadening, respectively [23], and these results will in turn degrade the 3-dB channel bandwidth [24]. While aforementioned works are mainly based on blue-green laser/LED under clear water condition. Nevertheless, the effects of such suspension particulates and dissolved organic matters requires further in-depth study. Xu et al. numerically studied the feasibility of enhancing the channel bandwidth by deploying red laser in a UWOC system mainly due to the smaller scattering effect in the longerwavelength [25]. Based on this study, Lee et al. experimentally demonstrated the performance enhancement by utilizing a near-infrared (NIR) laser, which shows the overall frequency response of the system gains an increment up to few tens of $\mathrm{MHz}$ with increasing particulates' concentration [26]. Besides mitigating the effect of suspension particulates, longer optical wavelengths also show the ability to mitigate the turbulence originated from temperature or salinity gradient. Oubei et al. experimentally measured the scintillation index for the red-green-lasers under turbulence, and red laser experiences much smaller signal fading as compared to the blue-green lasers [27].

On the other side, instead of using longer wavelength (red/NIR) to achieve high communication performance and to circumvent the above-mentioned scattering effect, it is feasible to construct a non-line-of-sight (NLOS) configuration by taking advantage of light scattering. In a NLOS underwater system, the field-of-view (FOV) for both transmitter and receiver are not directed towards each other. Rather, by scattering from the water molecular, suspension particles etc., the transmitter radiation will be redirected multiple times before arriving at the detector. Thus, NLOS communications relax the requirement of positioning, acquisition, and tracking (PAT). Sun et al. reported the first underwater ultraviolet $(375-\mathrm{nm})$ NLOS communication system, and experimentally measured the effect of geometries, water turbidity, and wavelengths based on path loss measurements [28]. They demonstrated that NLOS UWOC link can be significantly enhanced using the ultraviolet (375-nm) laser. It holds promises for circumventing the problems of scintillation, deep-fade, complete signal blockage in LOS UWOC.
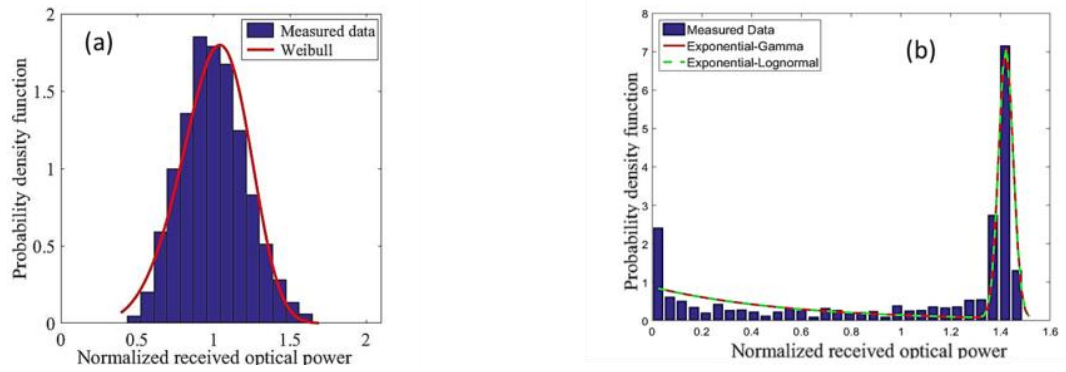

Fig. 3 UWOC turbulence related studies: (a) Weibull fitting distribution and the corresponding measured data histogram for a salinity gradient of $5 \times 10^{-2} \mathrm{~g} \cdot \mathrm{L}^{-1} \cdot \mathrm{cm}^{-1}$. (b) the histogram of the measured data along with the exponential-Gamma and exponential log-normal model for fresh water for a bubble level equal to $7.1 \mathrm{~L} / \mathrm{min}$.

In fact, a channel model is necessary to reflect the realistic performance evaluation of UWOC systems. Factors affecting such UWOC performance can be classified into two categories - inherent optical properties (IOPs) and apparent optical properties (AOPs). IOPs refer to the water medium related properties and do not rely on the light field geometry. The two fundamental IOPs are absorption and volume scattering function (VSF) from which the scattering coefficient can be derived [29]. The behavior of light propagation affected by IOPs can be modelled by radiative transfer equation (RTE) [30]. AOPs, on the other hand, include the properties that depend on both the medium and light field structure such as collimation and diffusion [31]. While the majority of studies for UWOC channel models focus on characterizing the absorption and scattering processes, the model to describing the channel performance in the presence of turbulence introduced by temperature or salinity gradient, should also be emphasized. Yi et al. proposed to use log-normal distribution, which has already been used to represent turbulenceinduced signal fading in free-space under weak turbulence, to characterize the weak oceanic turbulence with the probability density function [32]. Two more advanced models called Weibull distribution and Generalized Gamma Distribution (GGD) are found to be more suitable to model the statistical behavior of the measured irradiance for salinity [33] and temperature [34] induced turbulence, respectively. In oceans, bubbles can also affect UWOC performance, and this can be modelled by exponential-Gamma model [35]. Also, Oubei et al. demonstrated the 
feasibility of turbulence mitigation by adopting beam expansion [35]. Fig. 3(a) illustrates the Weibull fitting distribution and the corresponding measured data for a salinity gradient of $5 \times 10^{-2} \mathrm{~g} \cdot \mathrm{L}^{-1} \cdot \mathrm{cm}^{-1}$, and Fig. 3(b) shows the histogram of the measured data along with the exponential-Gamma and exponential log-normal model for fresh water for a bubble level equal to $7.1 \mathrm{~L} \cdot \mathrm{min}^{-1}$. We have thus verified the performance of UWOC system in both uniform and turbulent channels. A review of the recent advances in UWOC link were also published in [36].

\section{References}

[1] T. C. Wu et al., "Blue laser diode enables underwater communication at 12.4 Gbps," Sci. Rep. 7, 40480 (2017).

[2] S. Karp, "Optical communications between underwater and above surface (satellite) terminals," IEEE Trans. Commun., 24(1), 66-81 (1976).

[3] J. B. Snow et al., "Underwater propagation of high data rate laser communications pulses," Proc. SPIE 1750, 419-427 (1992).

[4] F. Hanson and S. Radic, "High bandwidth underwater optical communication," Appl. Opt. 47, 277-283 (2008).

[5] P. Tian et al., "High-speed underwater optical wireless communication using a blue GaN-based micro-LED," Opt. Express 25(2), 1193-1201 (2017).

[6] Y. F. Huang et al., "Filtered Multicarrier OFDM Encoding on Blue Laser Diode for 14.8-Gbps Seawater Transmission," J. Lightw. Technol., 36(9), 1739-1745 (2018).

[7] K.-T. Ho et al., "3.2 Gigabit-per-second Visible Light Communication Link with InGaN/GaN MQW Micro-photodetector," Opt. Express 26(3), 3037-3045 (2018).

[8] C. Shen et al., "Semipolar InGaN quantum-well laser diode with integrated amplifier for visible light communications," Opt. Express 26(6), A219-A226 (2018).

[9] H. M. Oubei et al., "2.3 Gbit/s underwater wireless optical communications using directly modulated 520 nm laser diode," Opt. Express 23(16), 20743-20748 (2015).

[10] C.-Y. Li et al.,"16 Gb/s PAM4 UWOC system based on 488-nm LD with light injection and optoelectronic feedback techniques," Opt. Express, 25(10), 11598-11605(2017).

[11] C. Shen et al., "20-meter underwater wireless optical communication link with 1.5 Gbps data rate," Opt. Express 24(22), 25502-25509 (2016).

[12] Y. Chen et al.,"26 m/5.5 Gbps air-water optical wireless communication based on an OFDM-modulated 520-nm laser diode,” Opt. Express 25(13), 14760-14765 (2017).

[13] X. Liu et al., "34.5 m underwater optical wireless communication with 2.70 Gbps data rate based on a green laser diode with NRZ-OOK modulation," Opt. Express 25(22), 27937-27947 (2017).

[14] J. B. Snow et al., "Underwater propagation of high-data rate laser communications pulses," Proc. SPIE 1750, 419-427 (1992).

[15] H. M. Oubei et al., "4.8 Gbit/s 16-QAM-OFDM transmission based on compact 450-nm laser for underwater wireless optical communication," Opt. Express 23(18), 23302-23309 (2015).

[16] K. Nakamura, I. Mizukoshi, and M. Hanawa, "Optical wireless transmission of 405 nm, 1.45 Gbit/s optical IM/DD-OFDM signals through a 4.8 m underwater channel," Opt. Express 23(2), 1558-1566 (2015).

[17] H. M. Oubei et al., "Wireless optical transmission of $450 \mathrm{~nm}, 3.2$ Gbit/s 16-QAM-OFDM signals over $6.6 \mathrm{~m}$ underwater channel," in Conference on Lasers and Electro-Optics (CLEO), pp. SW1F.1 (2016).

[18] J. Xu et al., "Underwater wireless transmission of high-speed QAM-OFDM signals using a compact red-light laser," Opt. Express 24(8), 8097-8109 (2016).

[19] J. Xu et al., "Directly modulated green-light diode-pumped solid-state laser for underwater wireless optical communication," Opt. Lett. 42(9), 1664-1667 (2017).

[20] T. C. Wu et al., "Blue laser diode enables underwater communication at 12.4 Gbps," Sci. Rep. 7, 40480 (2017).

[21] H. H. Lu et al., “An 8 m/9.6 Gbps underwater wireless optical communication system,” IEEE Photon. J. 8(5), 7906107 (2016).

[22] A. Al-Halafi et al., "Real-time video transmission over different underwater wireless optical channels using a directly modulated 520 nm laser diode" IEEE/OSA J. Opt. Commun. Netw. 9(10), 826-832 (2017).

[23] C. D. Mobley, "The Optical Properties of Water" in Handbook of optics, M. Bass, ed. (McGraw-Hill, 1995).

[24] Z. Q. Zeng et al., "A survey of underwater optical wireless communications," IEEE Commun. Surveys Tuts. 19(1), 204-238 (2016).

[25] J. Xu et al., "Underwater wireless transmission of high-speed QAM-OFDM signals using a compact red-light laser," Opt. Express 24(8), 8097-8109 (2016).

[26] I. E. Lee et al., "Bandwidth enhancement of wireless optical communication link using a near-infrared laser over turbid underwater channel," in 2017 Conference on Lasers and Electro-Optics Pacific Rim (CLEO-PR), pp. 1-5 (2017).

[27] H. M. Oubei et al., "Scintillations of RGB laser beams in weak temperature and salinity-induced oceanic turbulence," in Underwater Communication and Networking (UComm), accepted (2018).

[28] X. Sun et al., "375-nm ultraviolet-laser based non-line-of-sight underwater optical communication," Opt. Express 26(10), 12870-12877 (2018).

[29] N. G. Jerlov and F. F. Koczy, Photographic Measurements of Daylight in Deep Water (Elanders boktr., 1951).

[30] S. Arnon, J. Barry and G. Karagiannidis, Advanced Optical Wireless Communication Systems (Cambridge University Press, 2012).

[31] L.J. Johnson, F. Jasman,R. J. Green, and M. S. Leeson, "Recent advances in underwater optical wireless communications," J. Underw. Technol., 32(3), 167-175 (2014).

[32] X. Yi, Z. Li, and Z. Liu, "Underwater optical communication performance for laser beam propagation through weak oceanic turbulence," Appl. Opt. 54(6), 1273-1278 (2015).

[33] H. Makine Oubei et al.," Efficient Weibull channel model for salinity induced turbulent underwater wireless optical communications," 12th Conference on Lasers and Electro-Optics Pacific Rim (CLEO-PR), p. 1 (2017).

[34] H. M. Oubei et al., "Simple statistical channel model for weak temperature-induced turbulence in underwater wireless optical communication systems," Opt. Lett., 42(13), 2455-2458 (2017).

[35] H. M. Oubei et al., "Performance Evaluation of Underwater Wireless Optical Communications Links in the Presence of Different Air Bubble Populations," IEEE Photon. J. 9(2), 1-9 (2017).

[36] H. M. Oubei et al., "Light based underwater wireless communication,” Jpn. J. Appl. Phys 57(8S2), 08PA06 (2018). 